\title{
Origin of Asymmetric Electric Double Layers at Electrified Oxide/ Electrolyte Interfaces
}

\author{
Mei Jia, Chao Zhang,* and Jun Cheng*
}

Cite This: J. Phys. Chem. Lett. 2021, 12, 4616-4622

Read Online

ABSTRACT: The structure of electric double layers (EDLs) dictates the chemistry and the physics of electrified interfaces, and the differential capacitance is the key property for characterizing EDLs. Here we develop a theoretical model for computing the differential Helmholtz capacitance $C_{\mathrm{H}}$ of oxide-electrolyte interfaces using density functional theory-based finite-field molecular dynamics simulations. It is found that the dipole of interfacial adsorbed groups (i.e., water molecule, hydroxyl ion, and proton) at the electrified $\mathrm{SnO}_{2}(110) / \mathrm{NaCl}$ interfaces significantly modulates the double layer potential which leads to the asymmetric distribution of $C_{\mathrm{H}}$. We also find that the dissociative water adsorption prefers the inner sphere binding of counterions, which in turn leads to a higher Helmholtz capacitance, compared with that of the nondissociative case at the interface. This work provides a molecular interpretation of asymmetric EDLs seen experimentally in a range of metal oxides/hydroxides.

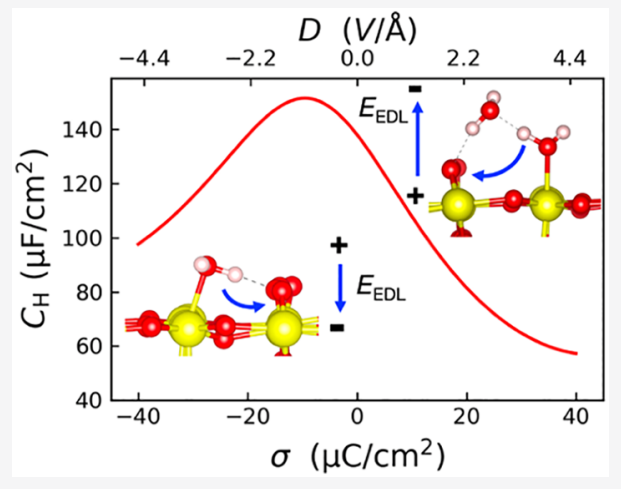

Se emiconducting oxide-electrolyte interfaces are highly electrified under working conditions. ${ }^{1,2}$ The charges of semiconducting oxide surfaces come from two sources. The difference in the electrochemical potentials between oxide and electrolyte leads to the depletion or accumulation of electrons between two phases and, therefore, the formation of the space charge layer. In addition, the adsorption of protons and hydroxyl groups generates positive charges and negative charges, respectively, at these oxide surfaces. ${ }^{3}$ The net interfacial proton charge is compensated by counterions from the electrolyte, which builds up the so-called protonic electric double layer (EDL),${ }^{4}$ which spans for just $3-5 \AA$, that is, the Helmholtz layer, at high ionic strength and under the flat band potential condition. ${ }^{4,5}$

EDLs play an important role in electrochemistry, ${ }^{6,7}$ photoelectrocatalysis, ${ }^{8}$ colloid science, and geochemistry. Some of the fundamental questions therein are the following: What are the surface composition and ions distribution in the EDL? How would adsorbed water molecules respond to the electric field? ${ }^{9}$ How would the dissociation and the recombination of interfacial water molecules affect the double layer potential $\Delta U$ ? What would the differential capacitance look like, that is, the change of the Helmholtz capacitance with respect to the surface charge density? ${ }^{10}$ In this regard, experimental methods such as X-ray photoelectron spectroscopy and X-ray standing wave provide the ion adsorption and the surface potential information; ${ }^{11,12}$ the sum-frequency generation spectroscopy probes the polarization of water at charged interfaces; ${ }^{13,14}$ and titration experiments reveal the change of surface charge density versus pH. ${ }^{15,16}$ Nevertheless, the analysis and extraction of this microscopic interfacial information on EDL is rather difficult if not impossible. ${ }^{17-19}$

This calls for density functional theory-based molecular dynamics simulation (DFTMD), which is a suitable computational method for describing the microscopic structure and the dynamics of water and ions near an interface. ${ }^{20,21}$ Recently, it has been shown that the finite-field DFTMD is a promising technique for modeling EDLs at electrified solid-liquid interfaces. $^{22-24}$ Finite-field DFTMD simulations relies on the constant electric displacement $D$ Hamiltonian introduced by Stengel, Spaldin, and Vanderbilt, ${ }^{25}$ and the corresponding expression of the average Helmholtz capacitance $C_{\mathrm{H}}$ is shown to $\mathrm{be}^{22}$

$$
C_{\mathrm{H}}=\frac{1}{2 \pi L_{z} A}\left(\frac{\partial q}{\partial\left\langle P_{z}\right\rangle}\right)_{D}
$$

where $L_{z}$ is the dimension of the supercell perpendicular to the interface, $q$ is the introduced surface proton charge, $A$ is the area of the $x, y$ cross section, and $\left\langle P_{z}\right\rangle$ indicates the ensemble averaged supercell polarization. Because $\left\langle P_{z}\right\rangle$ converges rapidly in the finite-field simulations, this makes $C_{\mathrm{H}}$ a new observable within the accessible time-scale of DFTMD. Note that in

Received: March 10, 2021

Accepted: May 5, 2021

Published: May 11, 2021 

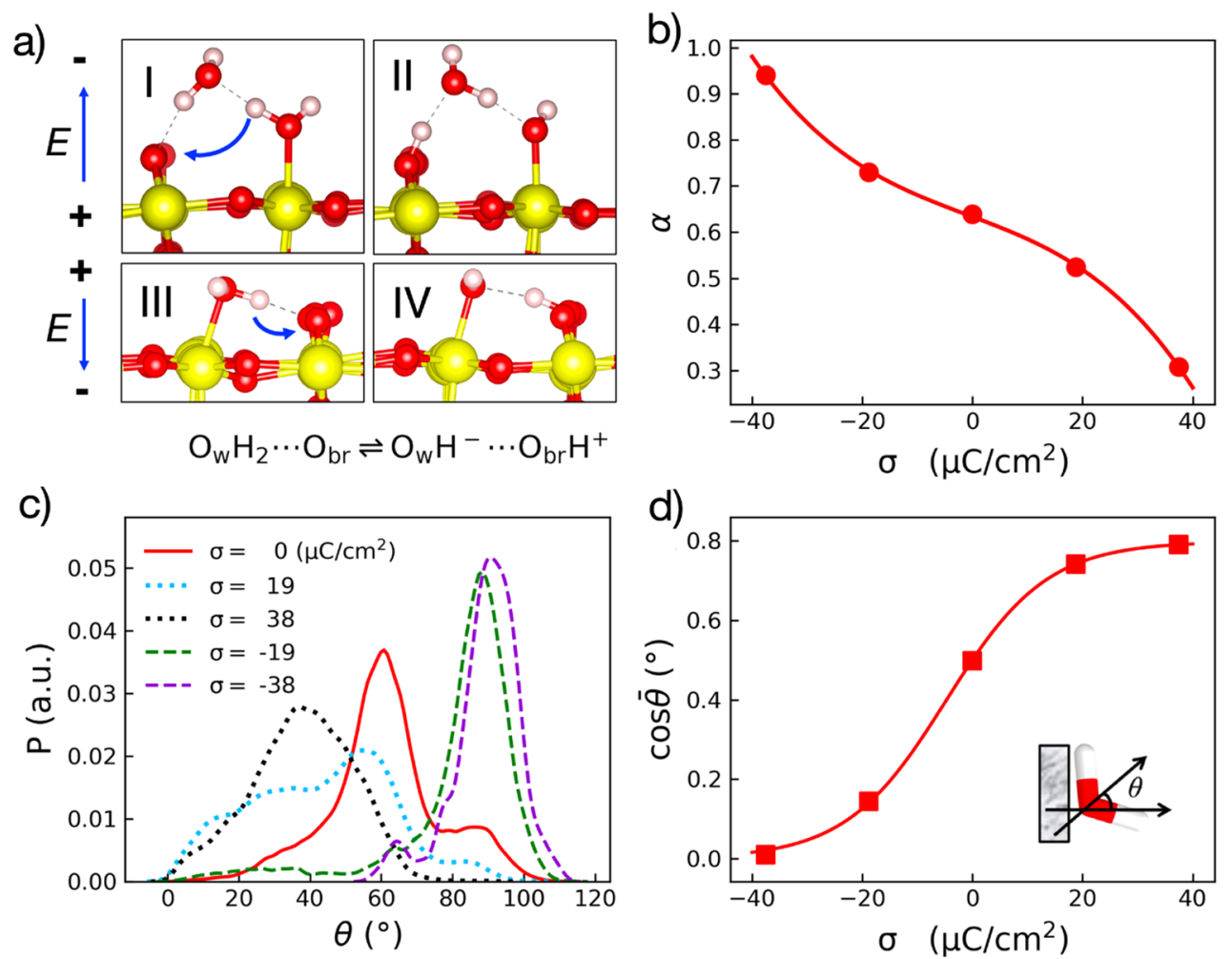

Figure 1. (a) Dissociation of interfacial water molecules at the low $\mathrm{pH}$ (I and II) and high pH (III and IV) interfaces in response to the electric field $\mathrm{E}$. The blue arrows on the left side show the direction of the electric field $\mathrm{E}$ of the double layer. (b) Plot of the degree of water dissociation $\alpha$ as a function of surface charge density $\sigma$. (c) Probability distribution of the angle $\theta$ between the bisector of adsorbed water molecules and surface normal for $\mathrm{SnO}_{2}(110) / \mathrm{NaCl}$ interfaces at different $\sigma$. (d) Plot of $\cos \bar{\theta}$ of adsorbed water molecules as a function of $\sigma$.

conjugation with eq 1 , two sides of the oxide slab take opposite types but the same amount of proton charges in the simulated supercell.

In the first work of applying finite-field DFTMD for modeling EDLs, $^{23}$ it was found that the Helmholtz capacitance at electrified rutile $\mathrm{TiO}_{2}(110) / \mathrm{NaCl}$ interfaces is much higher at high $\mathrm{pH}$ than that at low $\mathrm{pH}$ for the given surface charge density, and the interfacial proton transfer at low $\mathrm{pH}$ increases significantly the capacitance value. Compared with rutile $\mathrm{TiO}_{2}$, the isostructural cassiterite $\mathrm{SnO}_{2}$ has a characteristic dissociative water adsorption, ${ }^{26,27}$ which involves terminal adsorbed water $\mathrm{Sn}_{5 c} \mathrm{O}_{\mathrm{w}} \mathrm{H}_{2}$ to bridge oxygen site $\mathrm{Sn}_{2} \mathrm{O}_{\mathrm{br}}$ as

$$
\mathrm{Sn}_{5 \mathrm{c}} \mathrm{O}_{\mathrm{w}} \mathrm{H}_{2}+\mathrm{Sn}_{2} \mathrm{O}_{\mathrm{br}} \rightleftharpoons \mathrm{Sn}_{5 \mathrm{c}} \mathrm{O}_{\mathrm{w}} \mathrm{H}^{-}+\mathrm{Sn}_{2} \mathrm{O}_{\mathrm{br}} \mathrm{H}^{+}
$$

Then, the question naturally arises: how would the proton transfer affect the differential capacitance and vice versa? To answer that, herein the electrified $\mathrm{SnO}_{2}(110) / \mathrm{NaCl}$ electrolyte interfaces at different surface charge densities $\sigma$ were simulated with finite-field DFTMD, using the CP2K/Quickstep package $^{28,29}$ with the PBE functional. ${ }^{30}$ Detailed descriptions of the computational setup are given in the Supporting Information.

At low $\mathrm{pH}$, the $\mathrm{SnO}_{2}(110)$ surfaces are positively charged by adsorbing protons to $\mathrm{Sn}_{2} \mathrm{O}_{\mathrm{br}}$ sites, and the direction of dissociative proton transfer is opposite to that of the electric field $\mathrm{E}$ in the EDL (Figure 1a, I and II). At high $\mathrm{pH}$, the $\mathrm{SnO}_{2}(110)$ surfaces are negatively charged by the desorption proton from terminal $\mathrm{Sn}_{5 \mathrm{c}} \mathrm{O}_{\mathrm{w}} \mathrm{H}_{2}$, and the direction of the dissociative proton transfer is the same as that of the electric field $\mathrm{E}$ in the EDL (Figure 1a, III and IV). Because of this contrast, the degree of interfacial water dissociation (reaction 2) $\alpha$ increases with the $\mathrm{pH}$ in electrolyte (Figure $1 \mathrm{~b}$ ), when averaging the number of $\mathrm{Sn}_{5 c} \mathrm{O}_{\mathrm{w}} \mathrm{H}_{2}, \mathrm{Sn}_{5 \mathrm{c}} \mathrm{O}_{\mathrm{w}} \mathrm{H}^{-}, \mathrm{Sn}_{2} \mathrm{O}_{\mathrm{br}} \mathrm{H}^{+}$, and $\mathrm{Sn}_{2} \mathrm{O}_{\mathrm{br}}$ sites ( 16 sites in total on each surface) over DFTMD trajectories (see Supporting Information). Meanwhile, we find the free energy of water dissociation (Reaction 2) $\Delta A_{\text {diss }}$ decreases with the $\mathrm{pH}$ (see Supporting Information).

When looking at the orientation of interfacial water, it is found that the adsorbed water molecules point toward the electrolyte solution (Figure 1c), with the angle $\theta$ between the water dipole and surface normal of about $60^{\circ}$ at the PZC. This value is downshifted to $30^{\circ}-40^{\circ}$ and up-shifted to $80^{\circ}-90^{\circ}$, in response to the electric field in EDL. As shown in Figure 1d, the average angle of adsorbed water molecules shows a monotonic increment as a function of $\sigma$ in spite of a strong presence of the water dissociation.

It has long been known that the dipole of interfacial hydroxyl groups at oxide-electrolyte interfaces strongly affect the potential offset. $^{31-33}$ As shown in Figure 2a (see Supporting Information), the band edge is shifted upward with reference to the standard hydrogen electrode (SHE) scale $^{34}$ when going from the vacuum surface, to the water monolayer (ML) adsorption and the fully solvated $\mathrm{SnO}_{2}(110) / \mathrm{H}_{2} \mathrm{O}$ interface. This effect is also manifested when restraining water molecules adsorbed on the left side of the $\mathrm{SnO}_{2}$ slab not undergoing dissociation reactions at the PZC. In this case, interfacial water molecules would have different orientational distributions on the two sides of the $\mathrm{SnO}_{2}$ slab (Figure 2b), and the average $\theta$ of the left side (restrained) is about $10^{\circ}$ larger than that of the right side (free). This leads to a total net dipole pointing to the opposite of the surface normal (Figure 2c). As a consequence and illustrated in Figure 2d, the left side (restrained) of the slab has a higher potential than that of the right side. 

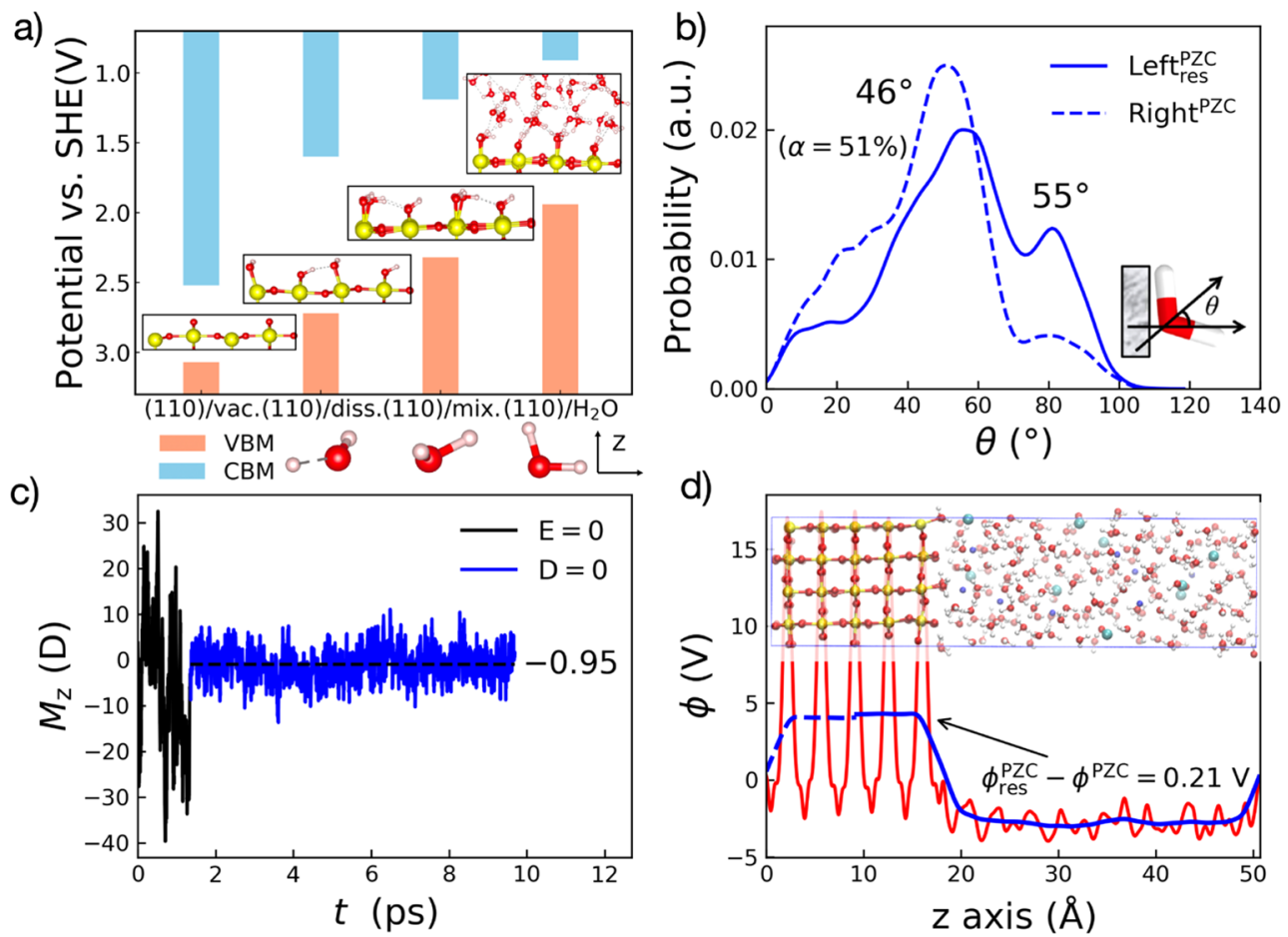

Figure 2. (a) Band alignment of $\mathrm{SnO}_{2}(110) /$ vacuum interface $((110) /$ vac. $)$, a monolayer of dissociatively adsorbed water interfaces $((110) /$ diss. $)$, mixed molecularly adsorbed and dissociatively adsorbed water interface $((110) /$ mix. $)$, and the $\mathrm{SnO}_{2}(110) / \mathrm{H}_{2} \mathrm{O}$ interface $\left((110) / \mathrm{H}_{2} \mathrm{O}\right)$ from left to right. The insets are the corresponding simulated models. (b) Probability distribution of the angle $\theta$ between the bisector of adsorbed water molecules and surface normal for the left side restrained ( Left $_{\text {res }}^{\mathrm{PZC}}$ ) and the right side of $\mathrm{SnO}_{2}(110) / \mathrm{NaCl}$ interface (Right ${ }^{\mathrm{PZC}}$ ) at PZC. "Restrained" means the $\mathrm{O}-\mathrm{H}$ bond of adsorbed water molecules were attached with a restraining potential to prevent the dissociation (see Computational setup). (c) Time evolution of the total dipole moment $M_{z}$ for left side restrained $\mathrm{SnO}_{2}(110) / \mathrm{NaCl}$ interface at PZC, when switching the electric boundary condition. Black dashed line is the time average of $M_{z}$. (d) The electrostatic potential $\phi$ profile at PZC. $\phi_{\text {res }}^{\mathrm{PZC}}$ and $\phi^{\mathrm{PZC}}$ are electrostatic potentials for left restrained side and right side.

On the basis of these observations made in Figure 1 and 2, we have formulated a theoretical model of differential Helmholtz capacitance at oxide-electrolyte interfaces to take the water dipole contribution into account explicitly. We begin with the textbook definition of the capacitance $C_{\mathrm{H}}$

$$
\frac{1}{C_{\mathrm{H}}}=\frac{\partial U}{\partial \sigma}
$$

where $U$ is the potential drop across the Helmholtz layer, and $\sigma$ is the surface charge density.

Then, applying the fundamental relation $D=E+4 \pi P$ and using the field expressions instead of potential and charge density, we can rewrite the above expression as

$$
\begin{aligned}
\frac{1}{C_{\mathrm{H}}} & =4 \pi l_{\mathrm{H}} \frac{\partial E}{\partial D} \\
& =4 \pi l_{\mathrm{H}} \frac{\partial\left(D-4 \pi P^{\mathrm{PZC}}-4 \pi \Delta P\right)}{\partial D} \\
& =4 \pi \frac{l_{\mathrm{H}}}{\epsilon_{\mathrm{H}}{ }^{\circ}}-16 \pi^{2} l_{\mathrm{H}} \frac{\partial \Delta P(D)}{\partial D} \\
& =\frac{1}{C_{\mathrm{H}}{ }^{\circ}}-16 \pi^{2} l_{\mathrm{H}} \frac{\partial \Delta P(D)}{\partial D}
\end{aligned}
$$

where $l_{\mathrm{H}}$ is the width of the Helmholtz layer, $\epsilon_{\mathrm{H}}{ }^{\circ}$ is the corresponding interfacial dielectric constant. $P^{\mathrm{PZC}}$ is the total polarization at the $\mathrm{PZC}$, and $\Delta P=P-P^{\mathrm{PZC}}$, which contains the difference in the polarization with respect to PZC.
From the equation above, it is clear that we will have a constant term $C_{\mathrm{H}}{ }^{\circ}$, which is independent of $D$, and a water polarization term $\Delta P(D)$, which is a function of $D$.

In case we are using the polarization of adsorbed surface $\mathrm{O}-\mathrm{H}$ groups $\Delta P_{\mathrm{w}}$ as a descriptor, the $\Delta P_{\mathrm{w}}$ includes the contribution of adsorbed water molecules in both molecular and dissociative forms. One may scale $\Delta P_{\mathrm{w}}$ by the dielectric constant of the double layer $\epsilon_{\mathrm{H}}{ }^{\circ}$ to account for the electrostatic screening. This recasts eq 7 into the following form:

$$
\begin{aligned}
\frac{1}{C_{\mathrm{H}}} & =\frac{1}{C_{\mathrm{H}}{ }^{\circ}}-16 \pi^{2} \frac{l_{\mathrm{H}}}{\epsilon_{\mathrm{H}}{ }^{\circ}} \frac{\partial \Delta P_{\mathrm{w}}(D)}{\partial D} \\
& =\frac{1}{C_{\mathrm{H}}{ }^{\circ}}-\frac{16 \pi^{2}}{A \epsilon_{\mathrm{H}}{ }^{\circ}} \frac{\partial \Delta M_{\mathrm{w}}(D)}{\partial D}
\end{aligned}
$$

where $\Delta M_{\mathrm{w}}(D)=\Omega \cdot \Delta P_{\mathrm{w}}(D)$, the volume $\Omega=A \cdot l_{\mathrm{H}}$. Here $\Delta M_{\mathrm{w}}(D)$ is the total dipole moment difference of interfacial groups (including the molecular and dissociative forms of adsorbed water) with respect to PZC. We can get $\Delta M_{\mathrm{w}}=\left(M_{\mathrm{w}}\right.$. $\left.N_{\mathrm{w}}-M_{\mathrm{w}}^{0} \cdot N_{\mathrm{w}}^{0}\right)+\left(M_{\mathrm{dis}} \cdot N_{\text {dis }}-M_{\mathrm{dis}}^{0} \cdot N_{\mathrm{dis}}^{0}\right) \cdot M_{\mathrm{w}}, M_{\mathrm{dis}}, N_{\mathrm{w}}$, and $N_{\text {dis }}$ are the dipole moment and number of adsorbed water molecule and dissociated $\left(\mathrm{OH}^{-}+\mathrm{H}^{+}\right)$groups (see Figure S5a). Note that in the model introduced here, there is only one free parameter $\epsilon_{\mathrm{H}}{ }^{\circ}$ in eq 9 . Instead, both $l_{\mathrm{H}}$ and $\Delta M_{\mathrm{w}}(D)$ can be determined from finite-field DFTMD simulations. This allows us to obtain the differential capacitance rather than applying the one-shot estimator (eq 1 ).

Then, we applied the eq 9 to fit $U-U^{\mathrm{PZC}}$, that is, the potential drop crossing the Helmholtz layer with respect to that at the 
a)

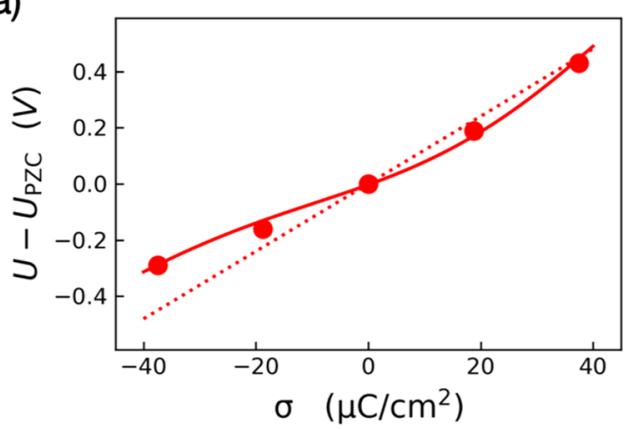

c)

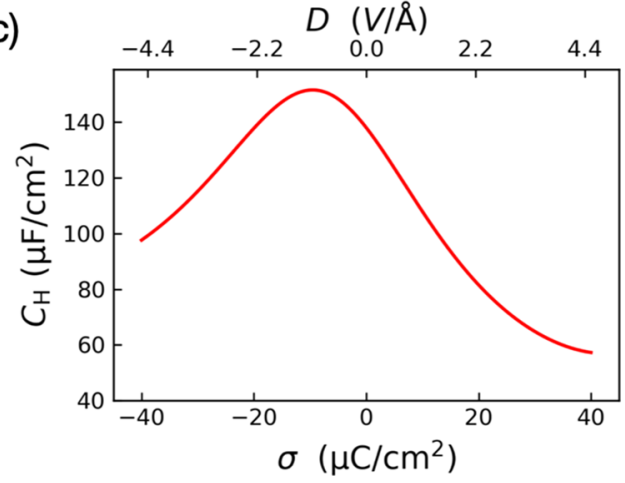

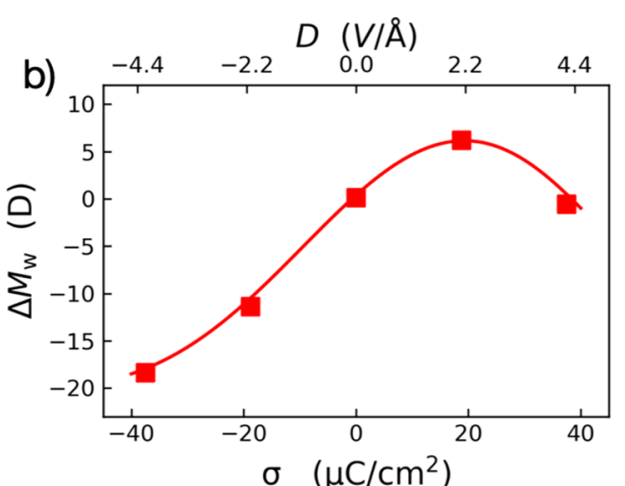

d)

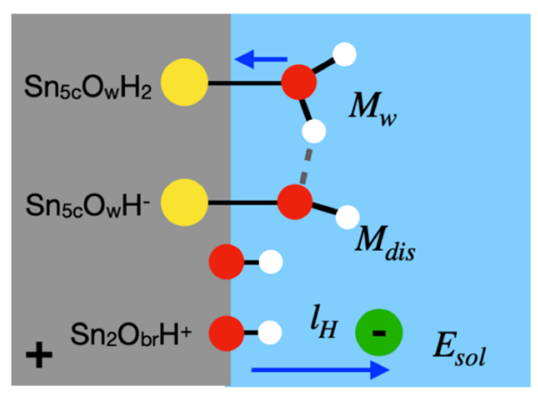

Figure 3. (a) Potential drop across the Helmholtz layer with respect to that at PZC. (b) Variation of the total dipole moment difference of interfacial groups (including the molecular and dissociative forms of adsorbed water) $\Delta M_{\mathrm{w}}(D)$ with respect to PZC. (c) The differential capacitance of the Helmholtz layer $C_{\mathrm{H}}$ as a function of surface charge density $\sigma .(\mathrm{d})$ A schematic illustration of the coupling between the EDL field and the water orientation/dissociation at the $\mathrm{SnO}_{2}(110) / \mathrm{NaCl}$ interface at low $\mathrm{pH}$.

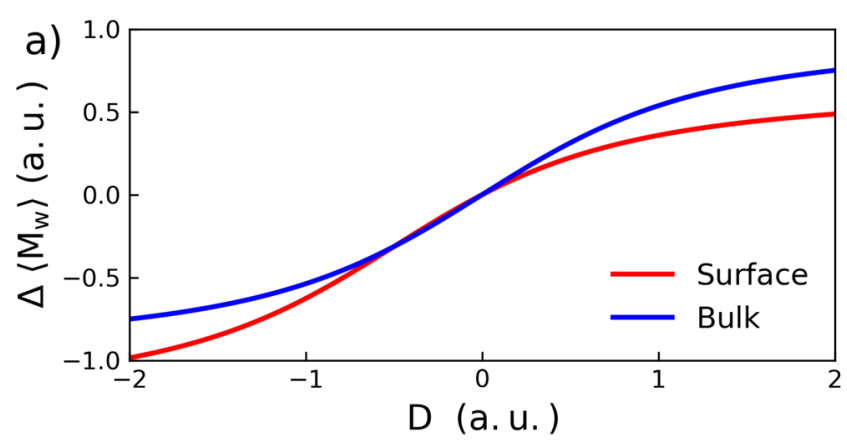

b)

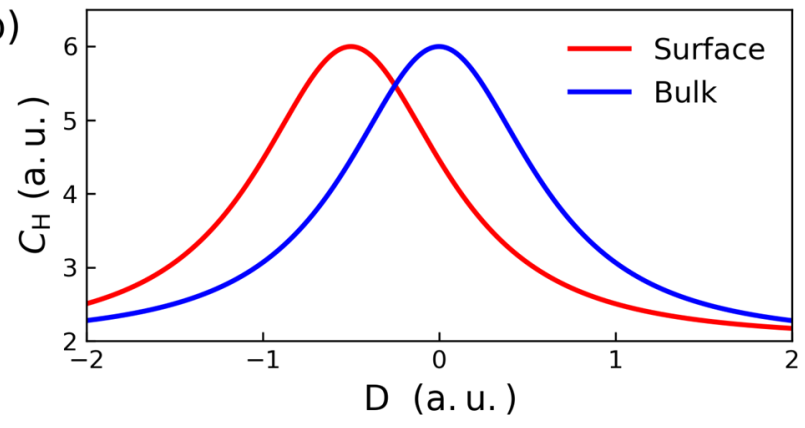

Figure 4. (a) Dependence of the dipole $\Delta\left\langle M_{\mathrm{w}}\right\rangle$ as a function of the electric displacement $D$ according to eq 10 . (b) The differential capacitance profile as a function of the electric displacement $D$ according to eq 11 .

PZC, which can be obtained from finite-field DFTMD trajectories using the macroscopic averaging technique ${ }^{35}$ (see Figure 3a and Figure S6a). In the case of $\mathrm{SnO}_{2}(110), \Delta M_{\mathrm{w}}(D)$ includes the contribution of adsorbed water molecules in both molecular and dissociative forms as shown in Figure $3 \mathrm{~b}, \mathrm{~d}$. The width of the Helmholtz layer $l_{\mathrm{H}}$ is about $2.6 \AA$, which estimates from the surface normal projected distance between the counterions and the nuclei of $\mathrm{O}_{\mathrm{br}}$ sites averaged over all surface charge densities.

The fitting result of $U(\sigma)-U^{\mathrm{PZC}}$ is given in Figure 3a, where the dashed line indicates the first constant term in eq 9. The free parameter $\epsilon_{\mathrm{H}}{ }^{\circ}$ turns out to be 24, which is quite close to the commonly assumed values for rutile structures. ${ }^{36}$ This agreement further justifies the theoretical model we formulated for the differential Helmholtz capacitance at oxide-electrolyte interfaces.

By taking the analytical derivative of $U(\sigma)-U^{\mathrm{PZC}}$, we obtain the differential capacitance $C_{\mathrm{H}}$ of the Helmholtz layer as shown in Figure 3c. We find that $C_{\mathrm{H}}$ shows a maximum of $\sim 151 \mu \mathrm{F} /$ $\mathrm{cm}^{2}$ at the negatively surface charge density $-10 \mu \mathrm{C} / \mathrm{cm}^{2}$, and it decreases to $\sim 100$ and $\sim 60 \mu \mathrm{F} / \mathrm{cm}^{2}$ when the surface charge density moves away to -40 and $40 \mu \mathrm{C} / \mathrm{cm}^{2}$, respectively. In addition, we find that $C_{\mathrm{H}}$ at the negatively charged surface is about $50 \%$ higher than that at the positively charged surface for the same value of $|\sigma|$, and this finding of asymmetric distribution of the differential $C_{\mathrm{H}}$ is in accord with what has been seen at electrified $\mathrm{TiO}_{2}(110) / \mathrm{NaCl}$ interfaces from finite-field DFTMD simulations $^{23}$ and in agreement with titration experiments of $\mathrm{SnO}_{2}$ at higher ionic strength. ${ }^{15,37}$ In fact, the asymmetric Helmholtz capacitance has been also seen in a range of metal oxides/hydroxides, such as $\mathrm{ZnO},{ }^{38} \mathrm{TiO}_{2},{ }^{16} \alpha-\mathrm{Al}_{2} \mathrm{O}_{3}{ }^{39}$ and $\gamma$ FeOOH. $^{40}$

This common feature observed in different oxides suggests that there should be a fundamental reason behind its cause. 
a) $\sigma=19 \mu \mathrm{C} / \mathrm{cm}^{2}$

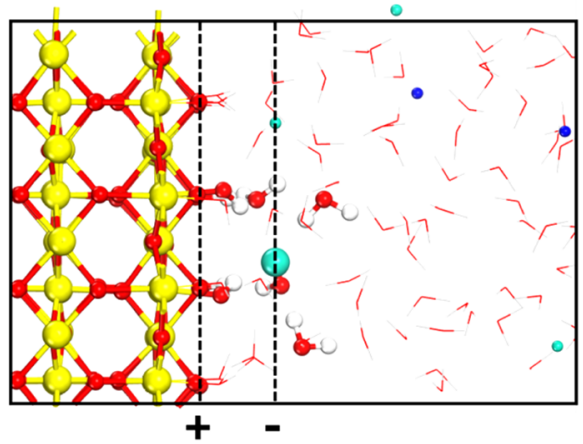

C) $\sigma=19 \mu \mathrm{C} / \mathrm{cm}^{2}$ (Res)

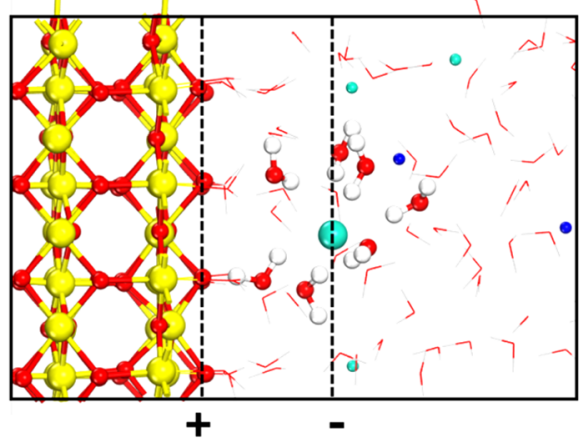

e) $\sigma=19 \mu \mathrm{C} / \mathrm{cm}^{2}$

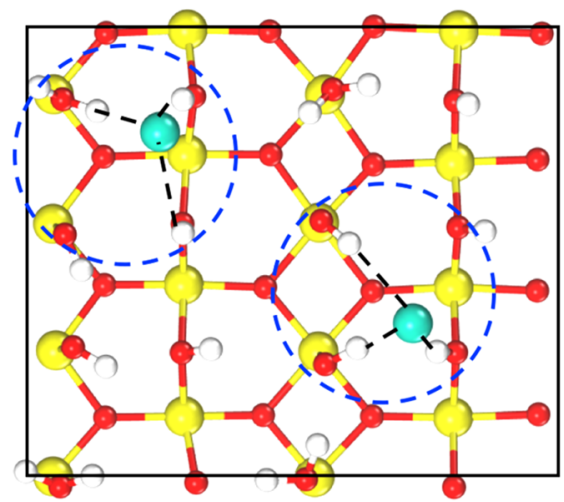

b) $\sigma=-38 \mu \mathrm{C} / \mathrm{cm}^{2}$

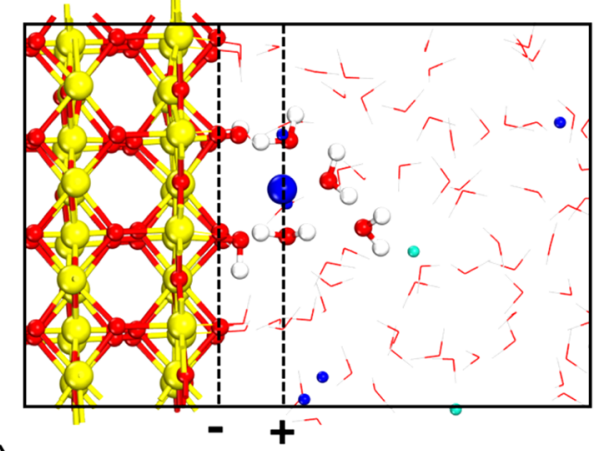

d) $\sigma=-38 \mu \mathrm{C} / \mathrm{cm}^{2}$ (Res)

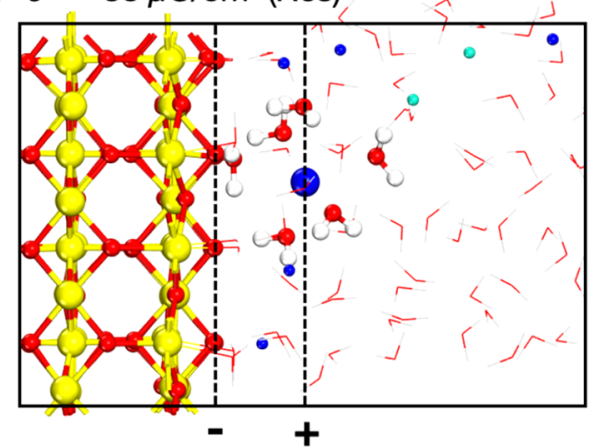

f) $\sigma=-38 \mu \mathrm{C} / \mathrm{cm}^{2}$

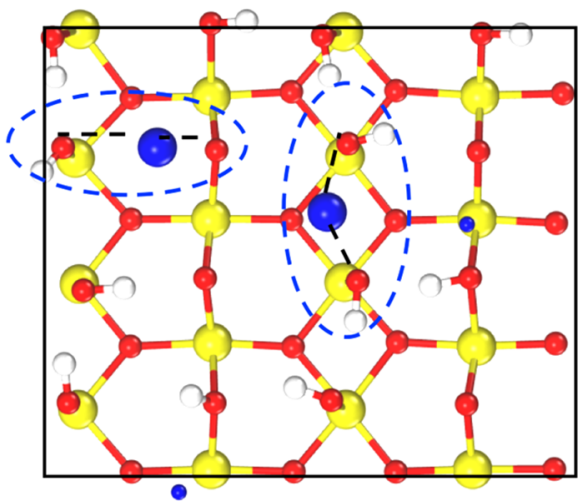

Figure 5. Side view $(\mathrm{a}-\mathrm{d})$ and top view $(\mathrm{e}, \mathrm{f})$ of the electrified $\mathrm{SnO}_{2}(110) / \mathrm{NaCl}$ electrolyte interface models at $\sigma=19$ and $-38 \mu \mathrm{C} / \mathrm{cm}^{2}$. Note that the adsorbed water molecules of (c) and (d) were restrained and not undergoing the dissociation. The $\mathrm{Sn}, \mathrm{O}, \mathrm{H}, \mathrm{Na}$, and $\mathrm{Cl}$ atoms were colored in yellow, red, white, blue, and cyan, correspondingly. The water molecules and surface $\mathrm{O}-\mathrm{H}$ groups coordinated to counterions were highlighted with the ballstick model.

Because the model given in eq 9 successfully captures the variation of $U(\sigma)-U^{\mathrm{PZC}}$ with $\Delta M_{\mathrm{w}}$ as the input, this points to the asymmetric orientation of adsorbed surface $\mathrm{O}-\mathrm{H}$ groups as the determining factor. At the metal oxide surfaces, water molecules have preferred orientations because of the chemisorption, where dipoles are pointing to the liquid phase, i.e. $\left\langle M_{\mathrm{w}}\right\rangle>0$ at PZC (Figure 1c). This surface effect can be captured by introducing an auxiliary field $D^{\mathrm{PZC}}$ into the Debye-type Hamiltonian $-\mu \cdot\left(D+D^{\mathrm{PZC}}\right) \cos (\theta)$, where $\mu$ is the dipole moment of noninteracting water molecules.

Then, one can show that the ensemble averaged $\Delta\left\langle M_{\mathrm{w}}\right\rangle$ with reference to the PZC (Figure $4 \mathrm{a}$ ) is

$$
\begin{aligned}
\Delta\left\langle M_{\mathrm{w}}\right\rangle= & \mu\left[\operatorname{coth}\left(\frac{\mu\left(D+D^{\mathrm{PZC}}\right)}{k T}\right)-\frac{k T}{\mu\left(D+D^{\mathrm{PZC}}\right)}\right] \\
& -\left.\left\langle M_{\mathrm{w}}\right\rangle\right|_{D=0}
\end{aligned}
$$

When taking the derivative of eq 10 with respect to $D$ and combining it with eq 7 , this leads to the expression of the differential capacitance $C_{\mathrm{H}}$ at the oxide surface (Figure $4 \mathrm{~b}$ ) as

$$
\begin{aligned}
\frac{1}{C_{\mathrm{H}}}= & \frac{1}{C_{\mathrm{H}}{ }^{\circ}}-\frac{16 \pi^{2}}{A} \frac{\mu^{2}}{k T}\left[1-\operatorname{coth}\left(\frac{\mu\left(D+D^{\mathrm{PZC}}\right)}{k T}\right)^{2}\right. \\
& \left.+\left(\frac{k T}{\mu\left(D+D^{\mathrm{PZC}}\right)}\right)^{2}\right]
\end{aligned}
$$

It is worth stressing that $D^{\mathrm{PZC}}$ is not due to the proton charge as in $D$ but an intrinsic property of metal oxide surfaces. Moreover, $D^{\mathrm{PZC}}$ is positive, as in the same direction of the water dipole at the PZC (Figure 1c). This offset $D^{\mathrm{PZC}}$ due to the specific orientation of adsorbed water at the PZC is the origin for the 
asymmetric electric doubles layers at oxide-electrolyte interfaces.

The final question is how the chemical specificity of surfaces comes into play. Clearly, the magnitude and the sign of $D^{\mathrm{PZC}}$ is system-dependent. More importantly, in the case of $\mathrm{SnO}_{2}(110)$, the interaction between dissociated $\mathrm{OH}^{-}, \mathrm{H}^{+}$and counterions in electrolyte is direct and strong, forming the inner sphere coordination. For example, at the positively charged interfaces, as shown in Figure 5a,e, the counterion $\mathrm{Cl}^{-}$is stabilized by two neighboring $\mathrm{Sn}_{2} \mathrm{O}_{\mathrm{br}} \mathrm{H}^{+}$and two $\mathrm{Sn}_{5 \mathrm{c}} \mathrm{O}_{\mathrm{w}} \mathrm{H}^{-}$groups. Similarly, at the negatively charged interfaces, the counterion $\mathrm{Na}^{+}$is stabilized by two neighboring $\mathrm{Sn}_{5 c} \mathrm{O}_{w} \mathrm{H}^{-}$or two $\mathrm{Sn}_{2} \mathrm{O}_{\text {br }}$ groups as shown in Figure 5b,f.

In contrast, the outer sphere coordination is preferred when restraining adsorbed water molecules not undergoing the dissociation (Figure $5 \mathrm{c}, \mathrm{d}$ ). Consequently, the average $\mathrm{C}_{\mathrm{H}}$ of $\mathrm{SnO}_{2}$ with dissociatively adsorbed water molecules is about 109 $\mu \mathrm{F} / \mathrm{cm}^{2}$, which is $45 \%$ larger than that of $\mathrm{SnO}_{2}$ with water molecules restrained to the molecular adsorption $\sim 61 \mu \mathrm{F} / \mathrm{cm}^{2}$ (Figure S7c). Overall, they indicate that theoretical models connecting the macroscopic property and the microscopy information are essential for interpreting the differential capacitance $C_{\mathrm{H}}$ of oxide-electrolyte interfaces.

In summary, through a combination of finite-finite DFTMD simulations and a theoretical model, the quantitative relationship between interfacial water orientation, proton transfer and differential capacitance at the charged water interface of $\mathrm{SnO}_{2}(110)$ was established. It has been shown that the general phenomenon of asymmetric electric double layers seen in a range of metal oxides/hydroxides can be explained by the specific orientation of chemisorbed water molecules at the point of zero charge.

\section{ASSOCIATED CONTENT}

\section{SI Supporting Information}

The Supporting Information is available free of charge at https://pubs.acs.org/doi/10.1021/acs.jpclett.1c00775.

Detailed descriptions of computational setup, the degree of water dissociation $\alpha$, the free energy of water dissociation $\Delta A_{\text {diss }}$, band edge alignment, double layer potentials and differential capacitance at electrified $\mathrm{SnO}_{2}(110) / \mathrm{NaCl}$ interfaces (PDF)

\section{AUTHOR INFORMATION}

\section{Corresponding Authors}

Chao Zhang - Department of Chemistry-Ångström Laboratory, Uppsala University, 75121 Uppsala, Sweden; (1) orcid.org/ 0000-0002-7167-0840; Email: chao.zhang@kemi.uu.se

Jun Cheng - State Key Laboratory of Physical Chemistry of Solid Surfaces, iChEM, College of Chemistry and Chemical Engineering, Xiamen University, Xiamen 361005, China; ○ orcid.org/0000-0001-6971-0797; Email: chengjun@ xmu.edu.cn

\section{Author}

Mei Jia - State Key Laboratory of Physical Chemistry of Solid Surfaces, iChEM, College of Chemistry and Chemical Engineering, Xiamen University, Xiamen 361005, China

Complete contact information is available at: https://pubs.acs.org/10.1021/acs.jpclett.1c00775

\section{Notes}

The authors declare no competing financial interest.

\section{ACKNOWLEDGMENTS}

M.J. thanks Xiamen University for the support for a research visit at Uppsala University. The simulations were performed on the resources provided by the Swedish National Infrastructure for Computing (SNIC) at PDC. J.C. is grateful for the funding support from the National Natural Science Foundation of China (Grant Nos. 21861132015, 21991151, 21991150, and 22021001). This project has received funding from the European Research Council (ERC) under the European Unions Horizon 2020 research and innovation programme (grant agreement No. 949012).

\section{REFERENCES}

(1) Ardizzone, S.; Trasatti, S. Interfacial properties of oxides with technological impact in electrochemistry. Adv. Colloid Interface Sci. 1996, 64, 173-251.

(2) Schmickler, W.; Santos, E. Interfacial Electrochemistry; Interfacial Electrochemistry; Springer: Berlin, 2010.

(3) Stumm, W. Chemistry of the Solid-Water Interface; Chemistry of the Solid-Water Interface; Wiley: New York, 1992.

(4) Nozik, A. J.; Memming, R. Physical Chemistry of SemiconductorLiquid Interfaces. J. Phys. Chem. 1996, 100, 13061-13078.

(5) Gerischer, H. Neglected problems in the $\mathrm{pH}$ dependence of the flatband potential of semiconducting oxides and semiconductors covered with oxide layers. Electrochim. Acta 1989, 34, 1005-1009.

(6) Salanne, M.; Rotenberg, B.; Naoi, K.; Kaneko, K.; Taberna, P. L.; Grey, C. P.; Dunn, B.; Simon, P. Efficient storage mechanisms for building better supercapacitors. Nat. Energy 2016, 1, 16070.

(7) Merlet, C.; Rotenberg, B.; Madden, P. A.; Taberna, P.-L.; Simon, P.; Gogotsi, Y.; Salanne, M. On the molecular origin of supercapacitance in nanoporous carbon electrodes. Nat. Mater. 2012, 11, 306-310.

(8) Ni, M.; Leung, M. K.; Leung, D. Y.; Sumathy, K. A review and recent developments in photocatalytic water-splitting using $\mathrm{TiO}_{2}$ for hydrogen production. Renewable Sustainable Energy Rev. 2007, 11, 401-425.

(9) Li, C.-Y.; Le, J.-B.; Wang, Y.-H.; Chen, S.; Yang, Z.-L.; Li, J.-F.; Cheng, J.; Tian, Z.-Q. In situ probing electrified interfacial water structures at atomically flat surfaces. Nat. Mater. 2019, 18, 697.

(10) Hiemstra, T.; Van Riemsdijk, W. H. On the relationship between charge distribution, surface hydration, and the structure of the interface of metal hydroxides. J. Colloid Interface Sci. 2006, 301, 1-18.

(11) Shchukarev, A.; Boily, J. F. XPS study of the hematite-aqueous solution interface. Surf. Interface Anal. 2008, 40, 349-353.

(12) Brown, M. A.; Abbas, Z.; Kleibert, A.; Green, R. G.; Goel, A.; May, S.; Squires, T. M. Determination of Surface Potential and Electrical Double-Layer Structure at the Aqueous Electrolyte-Nanoparticle Interface. Phys. Rev. X 2016, 6, 011007-12.

(13) Jena, K. C.; Covert, P. A.; Hore, D. K. The Effect of Salt on the Water Structure at a Charged Solid Surface: Differentiating Secondand Third-order Nonlinear Contributions. J. Phys. Chem. Lett. 2011, 2, 1056-1061.

(14) Ohno, P. E.; Saslow, S. A.; Wang, H.-F.; Geiger, F. M.; Eisenthal, K. B. Phase-referenced nonlinear spectroscopy of the $\alpha$-quartz/water interface. Nat. Commun. 2016, 7, 13587.

(15) Rosenqvist, J.; Machesky, M. L.; Vlcek, L.; Cummings, P. T.; Wesolowski, D. J. Charging properties of cassiterite $\left(\alpha-\mathrm{SnO}_{2}\right)$ surfaces in $\mathrm{NaCl}$ and $\mathrm{RbCl}$ ionic media. Langmuir 2009, 25, 10852-10862.

(16) Předota, M.; Machesky, M. L.; Wesolowski, D. J.; Cummings, P. T. Electric double layer at the rutile (110) surface. 4. effect of temperature and $\mathrm{pH}$ on the adsorption and dynamics of ions. J. Phys. Chem. C 2013, 117, 22852-22866.

(17) Gross, A.; Sakong, S. Modelling the electric double layer at electrode/electrolyte interfaces. Curr. Opin. Electrochem. 2019, 14, 1-6. 
(18) Gileadi, E. Problems in interfacial electrochemistry that have been swept under the carpet. J. Solid State Electrochem. 2011, 15, 13591371.

(19) Le, J.-B.; Fan, Q.-Y.; Li, J.-Q.; Cheng, J. Molecular origin of negative component of Helmholtz capacitance at electrified $\mathrm{Pt}(111) /$ water interface. Sci. Adv. 2020, 6, eabb1219.

(20) Cheng, J.; Sprik, M. The electric double layer at a rutile $\mathrm{TiO}_{2}$ water interface modelled using density functional theory based molecular dynamics simulation. J. Phys.: Condens. Matter 2014, 26, 244108.

(21) Pham, T.; Ping, Y.; Galli, G. Modelling heterogeneous interfaces for solar water splitting. Nat. Mater. 2017, 16, 401-408.

(22) Zhang, C.; Sprik, M. Finite field methods for the supercell modeling of charged insulator/electrolyte interfaces. Phys. Rev. B: Condens. Matter Mater. Phys. 2016, 94, 245309.

(23) Zhang, C.; Hutter, J.; Sprik, M. Coupling of Surface Chemistry and Electric Double Layer at $\mathrm{TiO}_{2}$ Electrochemical Interfaces. J. Phys. Chem. Lett. 2019, 10, 3871-3876.

(24) Zhang, C.; Sayer, T.; Hutter, J.; Sprik, M. Modelling electrochemical systems with finite field molecular dynamics. J. Phys.: Energy 2020, 2, 032005.

(25) Stengel, M.; Spaldin, N. A.; Vanderbilt, D. Electric displacement as the fundamental variable in electronic-structure calculations. Nat. Phys. 2009, 5, 304-308.

(26) Kumar, N.; Kent, P. R. C.; Bandura, A. V.; Kubicki, J. D.; Wesolowski, D. J.; Cole, D. R.; Sofo, J. O. Faster proton transfer dynamics of water on $\mathrm{SnO}_{2}$ compared to $\mathrm{TiO}_{2}$. J. Chem. Phys. 2011, 134, 044706.

(27) Jia, M.; Zhang, C.; Cox, S. J.; Sprik, M.; Cheng, J. Computing Surface Acidity Constants of Proton Hopping Groups from Density Functional Theory-Based Molecular Dynamics: Application to the $\mathrm{SnO}_{2}(110) / \mathrm{H}_{2} \mathrm{O}$ Interface. J. Chem. Theory Comput. 2020, 16, 65206527.

(28) Hutter, J.; Iannuzzi, M.; Schiffmann, F.; VandeVondele, J. CP2K: atomistic simulations of condensed matter systems. Wiley Interdiscip. Rev.: Comput. Mol. Sci. 2014, 4, 15-25.

(29) VandeVondele, J.; Krack, M.; Mohamed, F.; Parrinello, M.; Chassaing, T.; Hutter, J. Quickstep: Fast and accurate density functional calculations using a mixed Gaussian and plane waves approach. Comput. Phys. Commun. 2005, 167, 103-128.

(30) Perdew, J. P.; Burke, K.; Ernzerhof, M. Generalized Gradient Approximation Made Simple. Phys. Rev. Lett. 1996, 77, 3865-3868.

(31) Han, F.; Zhou, Z.; Huang, Z.; Li, M.; Guo, L. Effect of Water Adsorption on Interfacial Structure and Band Edge Alignment of Anatase $\mathrm{TiO}_{2}(001) /$ Water by First-Principles Molecular Dynamics. J. Phys. Chem. C 2018, 122, 26965.

(32) Pham, T. A.; Lee, D.; Schwegler, E.; Galli, G. Interfacial Effects on the Band Edges of Functionalized Si Surfaces in Liquid Water. J. Am. Chem. Soc. 2014, 136, 17071-17077.

(33) Ping, Y.; Sundararaman, R.; Goddard, W. A., III Solvation effects on the band edge positions of photocatalysts from first principles. Phys. Chem. Chem. Phys. 2015, 17, 30499-30509.

(34) Le, J.-B.; Iannuzzi, M.; Cuesta, A.; Cheng, J. Determining Potentials of Zero Charge of Metal Electrodes versus the Standard Hydrogen Electrode from Density-Functional-Theory-Based Molecular Dynamics. Phys. Rev. Lett. 2017, 119, 016801.

(35) Junquera, J.; Cohen, M. H.; Rabe, K. M. Nanoscale smoothing and the analysis of interfacial charge and dipolar densities. J. Phys.: Condens. Matter 2007, 19, 213203.

(36) Sverjensky, D. A. Prediction of surface charge on oxides in salt solutions: Revisions f or 1:1 $\left(\mathrm{M}^{+} \mathrm{L}^{-}\right)$electrolytes. Geochim. Cosmochim. Acta 2005, 69, 225-257.

(37) Rodriguez-Santiago, V.; Fedkin, M. V.; Wesolowski, D. J.; Rosenqvist, J.; Lvov, S. N. Electrophoretic Study of the $\mathrm{SnO}_{2} /$ Aqueous Solution Interface up to $260^{\circ} \mathrm{C}$. Langmuir 2009, 25, 8101-8110.

(38) Blok, L.; de Bruyn, P. The ionic double layer at the $\mathrm{ZnO}$ solution interface: III. Comparison of calculated and experimental differential capacities. J. Colloid Interface Sci. 1970, 32, 533-538.
(39) Fitts, J. P.; Shang, X.; Flynn, G. W.; Heinz, T. F.; Eisenthal, K. B. Electrostatic surface charge at aqueous/alpha- $\mathrm{Al}_{2} \mathrm{O}_{3}$ single-crystal interfaces as probed by optical second-harmonic generation. J. Phys. Chem. B 2005, 109, 7981-6.

(40) Boily, J.-F. The variable capacitance model: a strategy for treating contrasting charge-neutralizing capabilities of counterions at the mineral/water interface. Langmuir 2014, 30, 2009-2018. 